\title{
TIME STEPPING SOLUTIONS OF THE TWO- DIMENSIONAL NONLINEAR WAVE RADIATION PROBLEM
}

\author{
G. X. Wu* and R. Eatock Taylor $\dagger$ \\ ${ }^{*}$ Department of Mechanical Engineering, University College London, Torrington Place, \\ London WC1E 7JE, U.K.; \\ †Department of Engineering Science, University of Oxford, Parks Road, Oxford OX1 3PJ, U.K.
}

(Received for publication 15 October 1994; accepted 30 November 1994)

\begin{abstract}
The two-dimensional nonlinear time domain free surface flow problem is analysed using potential flow theory. The problem is solved by a time marching method. At each time step two numerical approaches are used. One is based on the boundary element method in the complex plane. The complex potential is assumed to vary linearly within each element and the solution is obtained by imposing the boundary conditions at the nodes of the elements. The other approach is based on the finite element formulation. Triangular elements and linear shape functions are used. The solution is obtained by the Galerkin method. Numerical results are obtained for the wave elevation generated by a vertical wave maker. Results are also provided for a circular cylinder oscillating below the free surface. For these cases the finite element method is found to provide substantially more efficient computations than the boundary element method using equivalent discretizations.
\end{abstract}

\section{INTRODUCTION}

Wave loading on large volume offshore structures is commonly estimated based on linearized velocity potential theory. This assumes that the parameters under consideration, such as the wave amplitude, are small. Linearized theory has been found to be useful in many cases. In particular, it can capture important phenomena such as resonances at wave frequencies. However, neglect of all nonlinear terms in the linearized theory means that some important effects are missing. Most noticeable are slowly varying drift forces and the high frequency forces associated with "springing". These can be captured by a second-order analysis. In theory it is possible to improve further the prediction of wave loading by including third-, fourth- and higher-order terms. In practice, however, the mathematical problem beyond second order becomes increasingly unworkable. On the other hand, to ensure convergence of the perturbation series, the disturbances are required to be small. But it is loading in large waves which is most undesirable and has to be controlled. This requires fully nonlinear theory.

The fully nonlinear theory is usually solved by a time marching method. This assumes that the wave profile and the position of the structure are known at a particular instant. The problem can then be solved by numerical techniques. The Bernoulli equation enables us to find the force on the structure. If the structure is not fixed, Newton's law will give the new acceleration. The acceleration then gives a new velocity which further gives the new position of the structure. Similarly, the velocity obtained on the free surface will give a new free surface profile. All these will enable the problem to 
be solved at the next time step. The procedure can be repeated for any desired number of time steps.

The solution at each time step can be obtained by various numerical methods. Longuet-Higgins and Cokelet (1976) developed a boundary element method in the complex plane. This was later extended by several people (Vinje and Brevig, 1981; Lin et al., 1984; New et al., 1985; Zhao and Faltinsen, 1992) to permit solution of a variety of nonlinear problems. An alternative method based on a spatial finite difference formulation has been developed by Telste (1985) and Yeung and Vaidhanathan (1990). Recently, Wu and Eatock Taylor (1994) have developed a finite element formulation. Based on calculations for a wave maker started suddenly from rest, and a transient wave in a rectangular tank, it has been found that the finite element formulation can provide accurate results very efficiently.

In this paper, we shall consider the two-dimensional radiation problem. Two methods have been implemented to solve the potential problem at each time step: the boundary element method and the finite element method. The purpose is to compare the results obtained by two different methods for verification. A second objective is to assess the relative advantages of these methods for a particular problem. In the time domain problem, it is common that thousands of time steps are required. At each time step, the computational domain is discretized with thousands of nodes. It is extremely important to limit the CPU at each time step. A feature of these methods is that if one doubles the length of time $T$ over which the computation is made, the required CPU may be increased by tens of times. The reason is that, as $T$ increases, the accumulated error will increase. To maintain the same accuracy for the final solution, a smaller time step is needed, and more elements have to be used at each step, thus leading to a significant increase of CPU. This is why an efficient and accurate scheme at each time step is so important.

The paper is arranged as follows. The next section briefly summarizes the nonlinear formulation, and the following two sections provide the essential aspects of the two numerical methods used to solve the boundary-value problem at each time step (further details may be found in the above-mentioned publications). A characteristic of this problem is that, as time advances, an increasingly large domain needs to be included in the computation. It is shown in Section 5 how domain decomposition may be used in conjunction with the finite element method to avoid the difficulties which this might otherwise cause. Typical results are presented in Section 6, and some conclusions about the relative advantages of the methods are drawn in Section 7 .

\section{MATHEMATICAL FORMULATION}

We define a Cartesian coordinate system $O-x y$ such that the origin is on the mean free surface and $y$ points vertically upwards. The fluid is assumed to be incompressible and inviscid, and the flow is assumed to be irrotational. A velocity potential $\phi$ can then be introduced, which satisfies the Laplace equation

$$
\nabla^{2} \phi=0
$$

in the fluid domain $R$. On rigid surfaces (the body and the bottom), the boundary condition is 


$$
\frac{\partial \phi}{\partial \mathbf{n}}=F,
$$

where $F$ is the normal velocity on the body and is zero on the bottom, and $\mathbf{n}$ is the normal of the surface pointing out of the fluid domain. The boundary conditions on the free surface $S_{F}($ or $y=\eta$ ) can be written as

$$
\begin{aligned}
& g \eta+\frac{\partial \phi}{\partial t}+\frac{1}{2} \nabla \phi \cdot \nabla \phi=0, \\
& \frac{\partial \phi}{\partial y}=\frac{\partial \eta}{\partial t}+\frac{\partial \phi}{\partial x} \frac{\partial \eta}{\partial x},
\end{aligned}
$$

where $g$ is the gravitational acceleration and $t$ is time. Equations (3) may also be written in the Lagrangian form

$$
\begin{aligned}
& \frac{\mathrm{d} \phi}{\mathrm{d} t}=g \eta-\frac{1}{2} \nabla \phi \nabla \phi, \\
& \frac{\mathrm{d} x}{\mathrm{~d} t}=\frac{\partial \phi}{\partial x}, \quad \frac{\mathrm{d} y}{\mathrm{~d} t}=\frac{\partial \phi}{\partial y} .
\end{aligned}
$$

The initial conditions at $t=0^{+}$are given as

$$
\phi(x, y=\xi, t=0)=\chi(x) \quad \eta(x, t=0)=\xi(x) .
$$

We use a finite difference procedure to advance the solution in the time domain. Thus we assume that the potential on the free surface at time $t$ is $G$ (i.e. $G=\chi(x)$, when $t=0)$. Once the solution at time $t$ has been found, the potential on the new free surface profile at a subsequent time $t+\Delta t$ can be obtained using Equations (4) and (5). This provides the free surface condition at $t+\Delta t$, and the problem can be solved again.

\section{BOUNDARY ELEMENT METHOD}

The boundary element method is used to transform the differential equation in the fluid domain at each time step into an integral equation over the boundary. For the two-dimensional problem, a complex function $\beta$ is defined in the usual way with the potential being the real part and the stream function $\psi$ being the imaginary part. Cauchy's theorem then gives

$$
\oint \frac{\beta}{z-z_{0}} \mathrm{~d} z=0,
$$

where $z=x+i y$ and $z_{0}$ is a point outside the fluid domain. In our implementation, the boundary of the fluid domain is specified by $n$ nodes and the adjacent nodes are linked by straight lines. We assume that $\beta$ varies with $z$ linearly, or

$$
\beta=\sum_{j=1}^{n} \beta_{j} N_{j}(z),
$$

where 


$$
N_{j}(z)= \begin{cases}\left(z-z_{j+1}\right) /\left(z_{j}-z_{j+1}\right) & z \in\left(z_{j}, z_{j+1}\right) \\ \left(z-z_{j-1}\right) /\left(z_{j}-z_{j-1}\right) & z \in\left(z_{j-1}, z_{j}\right) \\ 0 & z \notin\left(z_{j-1}, z_{j+1}\right)\end{cases}
$$

and $\beta_{j}$ is the value of $\beta$ at node $j$. Substituting Equation (8) into (7) and letting $z_{0}$ approach node $z_{k}$, we have

$$
\sum_{j=1}^{n} A_{k j}\left(\phi_{j}+i \psi_{j}\right)=0
$$

where

$$
A_{k j}=\frac{z_{k}-z_{j-1}}{z_{j}-z_{j-1}} \ln \frac{z_{j}-z_{k}}{z_{j-1}-z_{k}}+\frac{z_{k}-z_{j+1}}{z_{j}-z_{j+1}} \ln \frac{z_{j+1}-z_{k}}{z_{j}-z_{k}} .
$$

From the boundary conditions we know either the potential or its normal derivative (hence the stream function). We define the surface on which the potential is specified as $S_{1}$ and the surface on which the strcam function is specificd as $S_{2}$. Thesc known values can then be moved to the right-hand side of Equation (10), leading to

$$
\left.\sum_{j=1}^{n_{2}} A_{k j} \phi_{j}\right|_{j \in S_{2}}+\left.i \sum_{j=1}^{n_{1}} A_{k j} \psi_{j}\right|_{j \in S_{1}}=-\left.\sum_{j=1}^{n_{1}} A_{k j} \phi_{j}\right|_{j \in S_{1}}+\left.i \sum_{j=1}^{n_{2}} A_{k j} \psi_{j}\right|_{j \in S_{2}},
$$

$n_{1}$ and $n_{2}$ are the number of nodes on $S_{1}$ and $S_{2}$ respectively. Furthermore, when node $k$ is on $S_{1}$ the real part of this equation is taken, and when node $k$ is on $S_{2}$ the imaginary part is taken. It should be noticed that on the intersection of the free surface and the body surface, both the potential and stream function are known and they are both moved to the right-hand side. The total number of equations is therefore the number of nodes minus the number of points on the intersection.

A particular advantage of using the complex potential here is that it leads to a diagonally dominated linear matrix equation at each time step. This allows Gauss-Seidel iteration to be used, requiring a CPU time of the order of the square of the number of nodes multiplied by the number of iterations to achieve the required degree of convergence. On the other hand, the real potential satisfies a mixed Dirichlet and Neumann problem at each time step, leading to a mixed Fredholm equation of first and second kinds. Such an equation is not always diagonally dominated and Gauss-Seidel itcration usually diverges. If, however, Gauss elimination is used instead, the CPU time is proportional to the cube of the number of nodes, and this will lead to a dramatic increase in CPU time when the number of nodes increases. Thus Equation (12) in the complex plane is far more efficient.

\section{FINITE ELEMENT METHOD}

The finite element method discretizes the problem in the entire modelled fluid domain $R$. This domain is enclosed by the body surface $S_{0}$, the free surface $S_{F}$, the bottom $S_{B}$, and an outer vertical boundary $S_{R}$. The velocity potential is writtten as

$$
\phi=\sum_{i=1}^{n} \phi_{j} N_{j}(x, y)
$$


where $\phi_{j}$ are the nodal values of the potential, $n$ is the number of the nodes and $N_{j}(x, y)$ are the shape functions. Application of the Galerkin method leads to

$$
\begin{aligned}
0 & =\int_{R} \nabla^{2} \phi N_{i} \mathrm{~d} R \\
& =\int_{R}\left[\nabla\left(\nabla \phi N_{i}\right)-\nabla \phi \nabla N_{i}\right] \mathrm{d} R \\
& =\int_{S} N_{i} \frac{\partial \phi}{\partial n} \mathrm{~d} S-\int_{R} \nabla \phi \nabla N_{i} \mathrm{~d} R,
\end{aligned}
$$

where $S$ is the boundary of $R$. By substituting the boundary conditions into the above equation, we obtain

$$
\left.\int_{R} \nabla N_{i} \sum_{j=1}^{n} \phi_{j} \nabla N_{j} \mathrm{~d} R\right|_{j \in S_{1}}=\left.\int_{R} \nabla N_{i} \sum_{j=1}^{n} \phi_{j} \nabla N_{j} \mathrm{~d} R\right|_{j \in S_{1}}+\int_{S_{2}} N_{i} F \mathrm{~d} S,
$$

where again $S_{1}$ and $S_{2}$ are, respectively, the surfaces on which the potential and its normal derivative are specified. The potential on the free surface is known from the free surface boundary condition and the corresponding terms have therefore been moved to the right-hand side of the equation. This scheme was adopted by $\mathrm{Wu}$ and Eatock Taylor (1994) and was found to be effective in dealing with the singularity at the intersection point between the free surface and the body surface. In matrix form, Equation (15) can be written as

$$
[A][\phi]=[B],
$$

where the coefficients are

$$
\begin{aligned}
A_{i j} & =\int_{R} \nabla N_{i} \nabla N_{j} \mathrm{~d} R \\
B_{i} & =\left.\int_{R} \nabla N_{i} \sum_{j=1}^{n} \phi_{j} \nabla N_{j} \mathrm{~d} R\right|_{j \in S_{F}}+\int_{S_{0}} N_{i} F \mathrm{~d} S .
\end{aligned}
$$

Here we have replaced $S_{2}$ by the body surface $S_{0}$, and $S_{1}$ by $S_{F}$ (on the assumption that $\partial \phi / \partial n=0$ on the outer vertical boundary $S_{R}$-this is further discussed in the next section). For the results obtained below, triangular elements have been used together with linear shape functions. The details of the formulation have been given by $\mathrm{Wu}$ and Eatock Taylor (1994).

One of the concerns in the time domain analysis is that the velocity has to be calculated accurately in order to avoid excessive accumulated error. A technique based on the Galerkin method has been proposed by Wu and Eatock Taylor, and has been found to be very satisfactory. Its disadvantage is that the velocity is obtained by solving another set of linear equations. The CPU time required is therefore doubled. On the other hand, the velocity is not needed everywhere. It is required only on the free surface (to calculate the position of the free surface and the potential on the free 
surface at the next time step) and on the body surface (to calculate the pressure). Thus for the results given below we have used the finite difference method to calculate the velocity, and this has been found to be satisfactory.

It is well known that the efficiency of the finite element formulation depends on the bandwidth of the final matrix equation, which in turn depends on the order in which the nodes are numbered. In this work, we have used an optimization scheme for numbering the nodes. Then, in each row, we retain only the coefficients within the local bandwidth. As the matrix is symmetric, we store only the upper half (in a onedimensional array). It is found that all these have improved the efficiency and memory requirement significantly.

\section{DOMAIN DECOMPOSITION}

As time increases, the radiated waves will propagate further away from the body. This means that the computational domain will become larger and larger. The computational domain may be truncated some distance away from the body, but this requires an appropriate condition (a radiation condition) to be imposed on the truncated boundary. For the fully nonlinear problem, however, such a condition has not been established for general purposes. There are various conditions currently in use but they all have their limitations. Here we have investigated an alternative.

If the computational domain becomes larger, the memory requirement will eventually exceed the practical limit that a computer system can provide. Thus we divide the computational domain into many small domains. The computation is then made in each domain. To demonstrate the principle of the method, we consider the two subdomains shown in Fig. 1.

The governing equations can be written as

$$
\nabla^{2} \phi_{k}=0 \quad k=1,2
$$

in the corresponding sub-domains;

$$
\frac{\partial \phi_{k}}{\partial n}=F_{k} \quad k=1,2
$$

on the body surface or the bottom of the fluid;

$$
\phi_{k}=G_{k} \quad k=1,2
$$

on the free surface. Thesc equations arc similar to those in Section 2. The difference

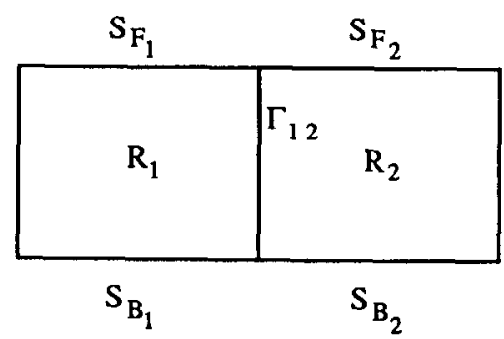

Fig. 1. Domain decomposition. 
is that as each potential $\phi_{k}$ is defined in only one sub-domain it is necessary to ensure continuity over the boundary of the sub-domains. This can be achieved by specifying

$$
\phi_{1}=\phi_{2}, \quad \frac{\partial \phi_{1}}{\partial n}=-\frac{\partial \phi_{2}}{\partial n}
$$

on $\Gamma_{12}$, where $n$ points out of the corresponding fluid domain.

To solve the above problem, an iterative procedure may be adopted. We use the following technique (Glowinski et al., 1984) which is started by choosing a function $\lambda^{0}$ so that

$$
\frac{\partial \phi_{1}^{0}}{\partial n}=-\frac{\partial \phi_{2}^{0}}{\partial n}=\lambda^{0}
$$

on $\Gamma_{12}$. (The superscript designates the iteration number.) This allows us to solve the problem in each sub-domain. We thereby obtain $\phi_{1}^{1}$ and $\phi \frac{1}{2}$ on $\Gamma_{12}$ corresponding to the first iteration. In the following steps we use

$$
\frac{\partial \phi_{1}^{m}}{\partial n}=-\frac{\partial \phi_{2}^{m}}{\partial n}=\lambda^{m}
$$

where

$$
\lambda^{m}=\lambda^{m-1}+\rho\left(\phi_{2}^{m-1}-\phi_{1}^{m-1}\right) .
$$

If $\rho$ is appropriately chosen, the iteration will converge. This has been shown by Glowinski et al. (1984), who have also given details of alternative ways of using domain decomposition.

\section{RESULTS AND DISCUSSION}

We first consider the problem of a vertical piston-type wave maker in water of depth $d$. The typical boundary element and finite element meshes are shown in Fig. 2(a) and (b). The motion of the wave maker is defined by the following equation:

(a)

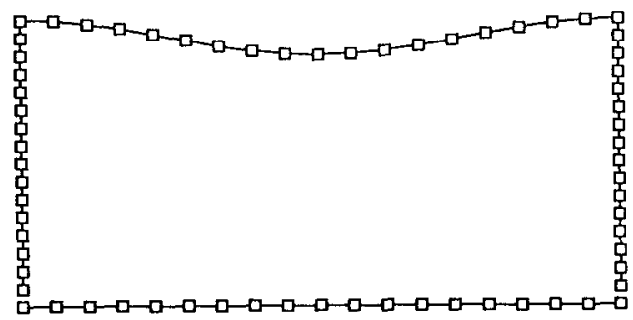

(b)

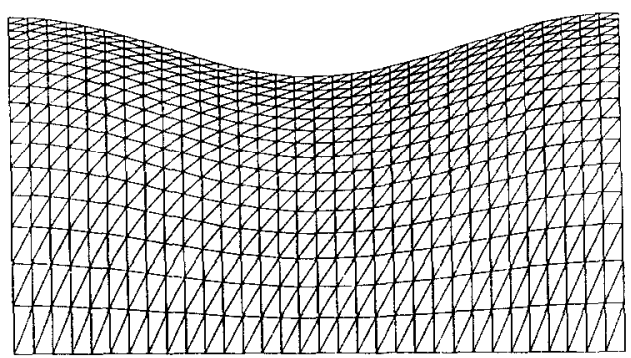

Fig. 2. Meshes for wave maker problem: (a) boundary elements; (b) finite elements. 


$$
\delta=\delta_{0}(1-\cos \omega t)
$$

The various parameters are nondimensionalized by redefining them as follows:

$$
\phi \rightarrow d(g d)^{\frac{1}{2}} \phi \quad(x, y) \rightarrow d(x, y) \quad t \rightarrow(d / g)^{\frac{1}{2}} t \quad \omega \rightarrow(g / d)^{\frac{1}{2}} \omega .
$$

It is then easy to confirm that the nondimensionalized boundary condition on the wave maker becomes

$$
\phi_{x}=F=\left(\omega \delta_{0} / d\right) \sin \omega t .
$$

Initially $G=0$ on the free surface and it is calculated from Equations (4) and (5) at subsequent time steps.

Figure 3 gives the results corresponding to $\omega=1.0$ and $\delta_{0} / d=0.1$. The dimensionless time step is taken as $\mathbf{0 . 0 2 5}$. Figure 3 (a) gives the wave elevation history on the wave maker, while Fig. 3(b) gives the wave elevation history at $x / d=12$. The computational domain is truncated at $x / d=40$ at which the fluid has not been significantly disturbed, as shown in Fig. 3(c). No domain decomposition was applied in the finite element analysis of this problem. The meshes are regenerated at each time step, and the values of the potential and the wave elevation at the nodes of each new mesh are obtained by interpolation.

It has been observed from the calculations based on the two methods that, as the number of nodes increases, the finite element method becomes superior both in terms of CPU and memory. Typically, the finite element method takes a few minutes on a DEC 3600 workstation for a mesh with 16 elements vertically times 640 horizontally (corresponding to a total of 10897 nodes); the boundary element method, however, with 16 nodes on the vertical and 640 on the horizontal surfaces (corresponding to a total of 1312 nodes), takes a few hours. Most interestingly, it has also been observed that as the number of nodes increases, the finite element method requires relatively less memory. This is because for the boundary element method all nodes are connected by the Green function, which leads to a full matrix. For the finite element method, however, the connections between the nodes are only through the physical links, which leads to a banded matrix. Thus, although the finite element method has far more nodes, the non-zero part of the coefficient matrix is smaller.

We next consider a submerged circular cylinder of radius $a$ oscillating periodically. The horizontal and vertical motions of the centre of the cylinder are defined by the following equations:

$$
\begin{aligned}
& \delta=\delta_{0}(1-\cos \omega t) \\
& \zeta=\zeta_{0}(1-\cos \omega t) .
\end{aligned}
$$

When the solution of the potential has been found, the forces on the cylinder can be found from the following equation (Newman, 1977):

$$
\mathbf{F}=-\rho \frac{\mathrm{d}}{\mathrm{d} t} \int_{S_{0}} \phi \mathbf{n d} S+\rho \int_{S_{0}}\left(\frac{\partial \phi}{\partial n} \nabla \phi-\frac{1}{2} \nabla \phi \nabla \phi \mathbf{n}\right) \mathrm{d} S .
$$

The parameters in this case are defined in a similar way as in Equation (24) apart from $d$ being replaced by $a$. Figure 4 gives the calculated results for purely horizontal 
Time stepping solutions of the nonlinear wave radiation problem
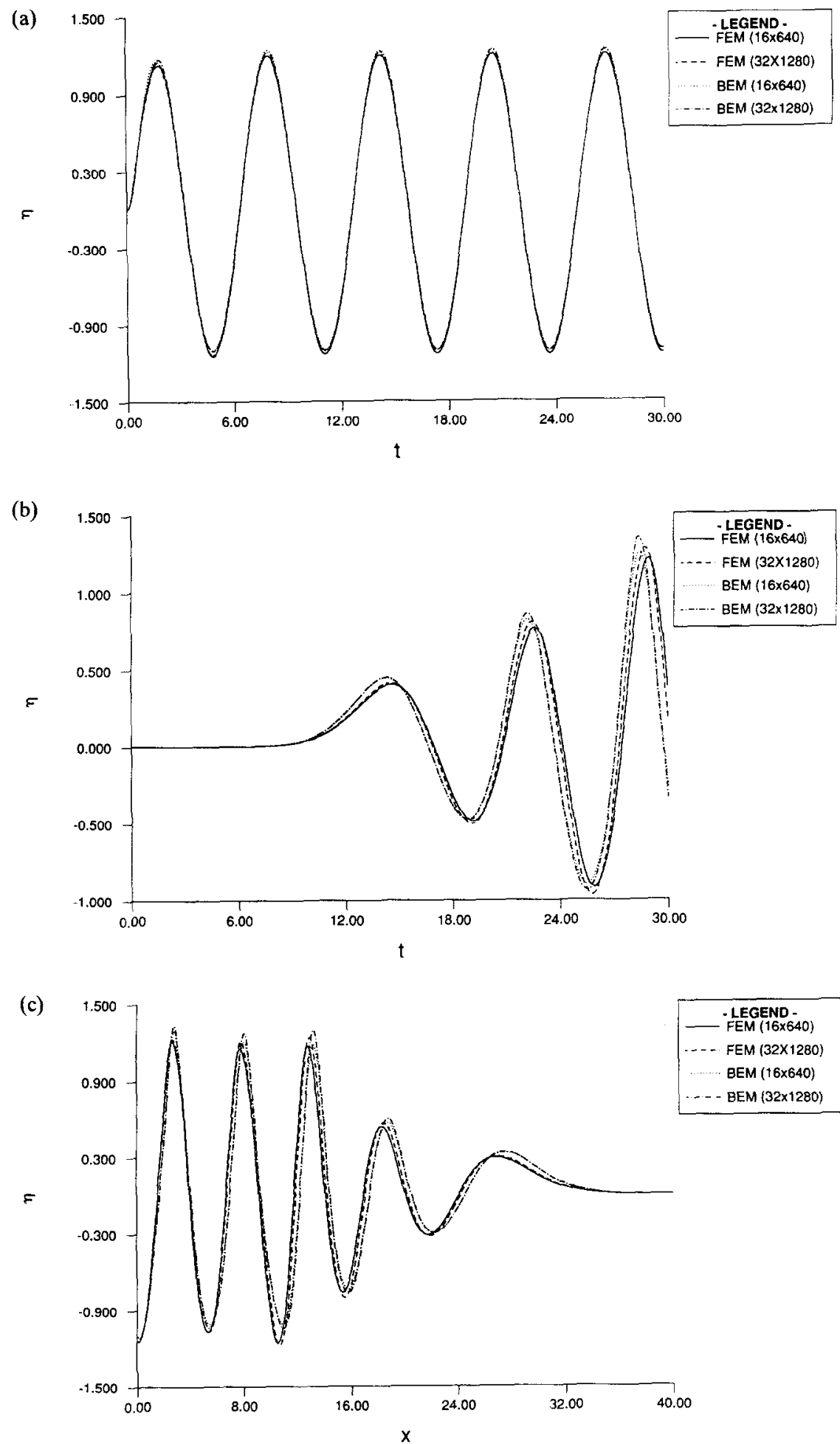

Fig. 3. Wave elevation time histories: (a) at the wave maker; (b) at $x / d=12$; (c) at $t=30$. 
(a)

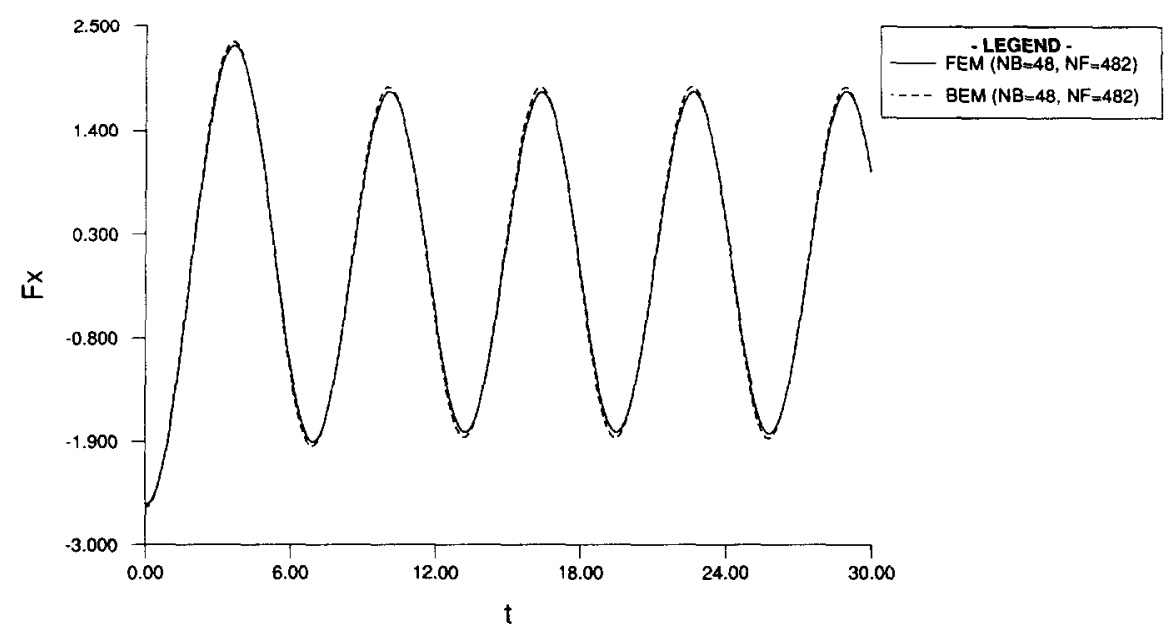

(b)

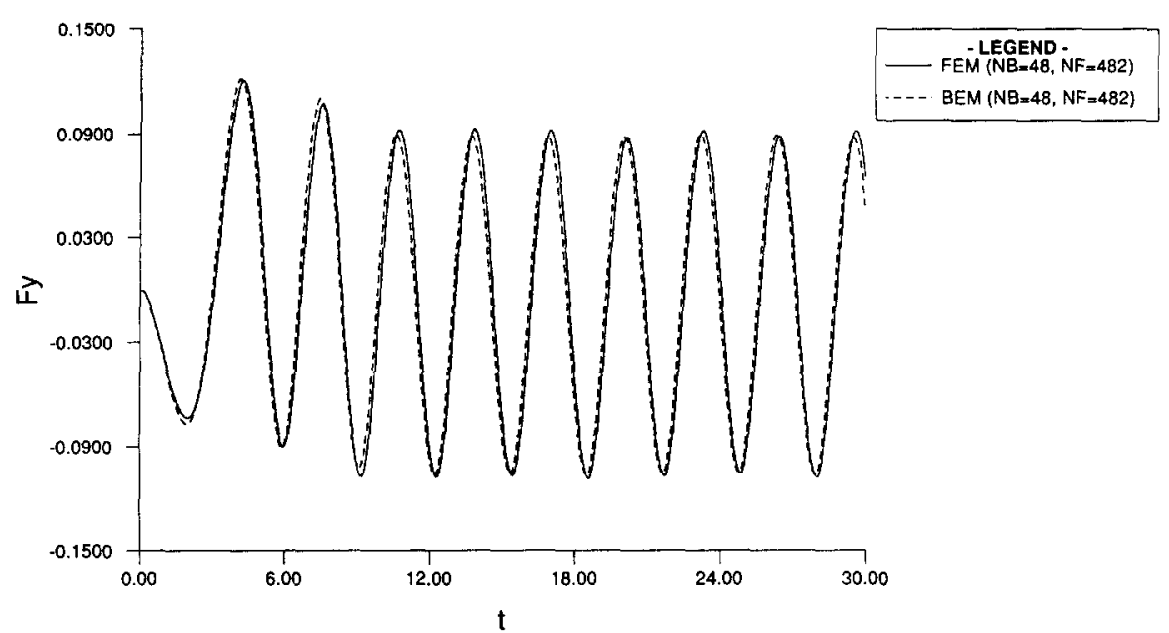

(c)

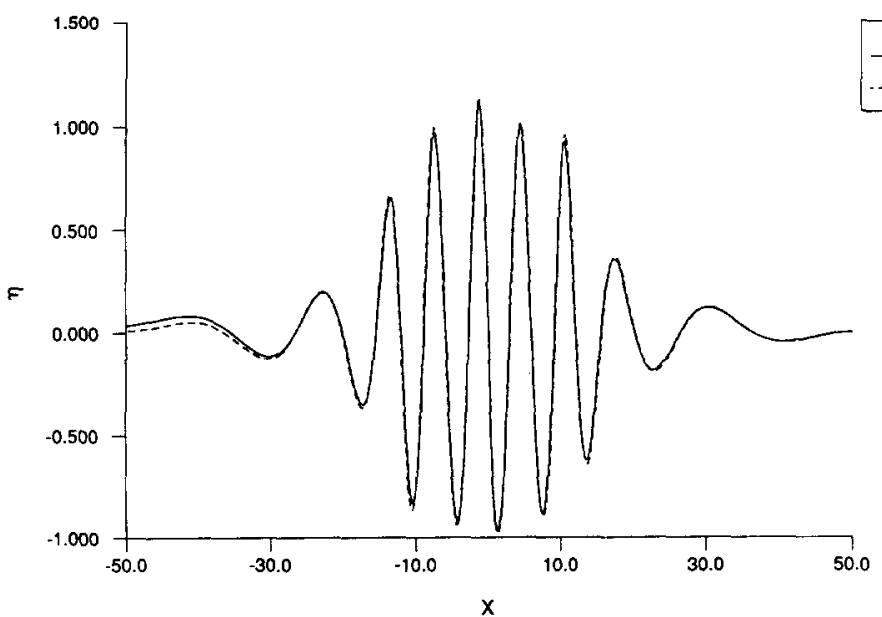

Fig. 4. Results for a submerged cylinder undergoing horizontal motion: (a) horizontal force; (b) vertical force; (c) wave elevation at $t=30$. 

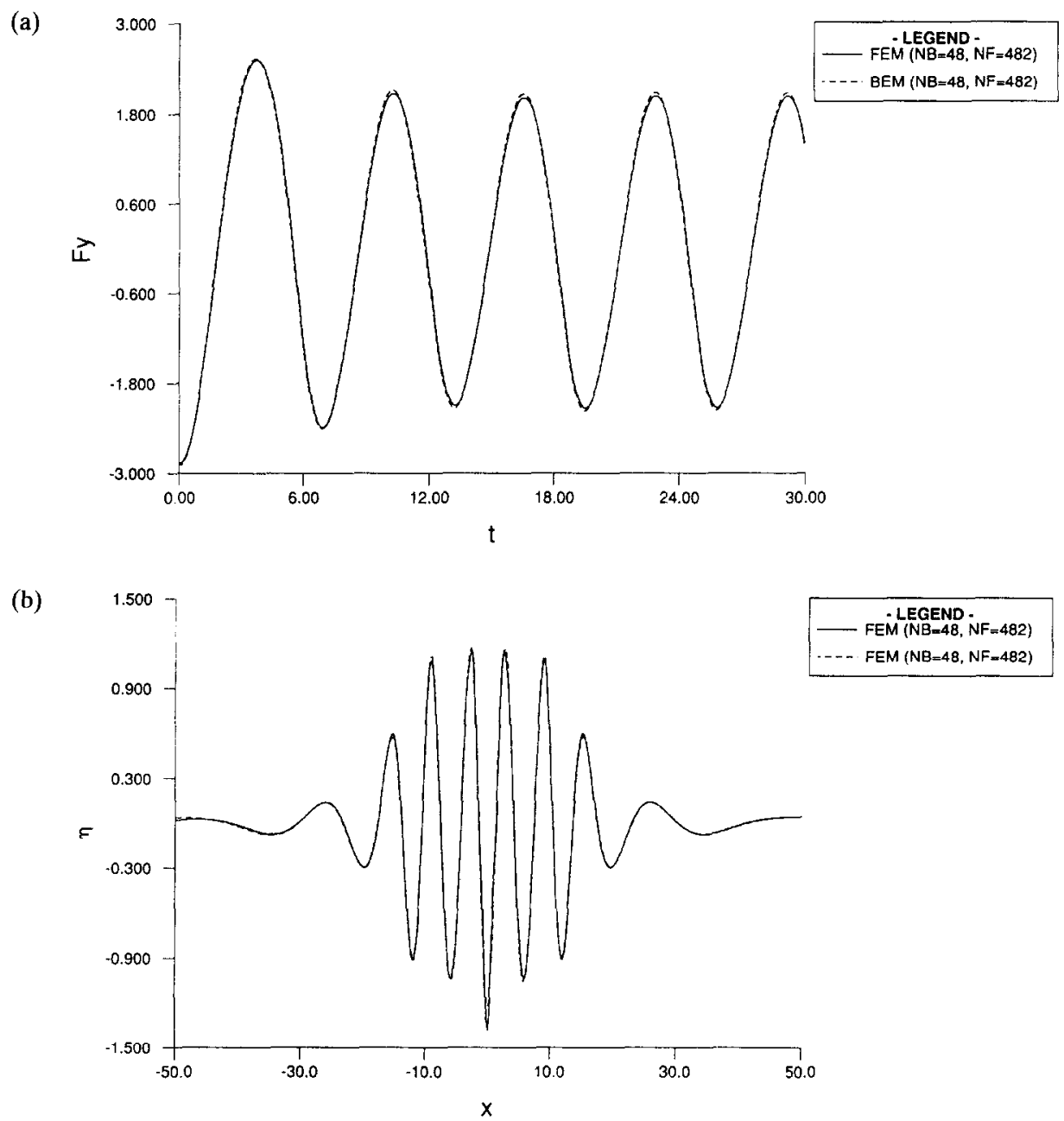

Fig. 5. Results for a submerged cylinder undergoing vertical motion: (a) vertical force; (b) wave elevation at $t=30$.

motion $(\zeta=0)$. NF and $N B$ indicate numbers of elements used on the free surface and body surface respectively. The dimensionless time step has been taken as 0.05 . The computational domain is truncated at $x= \pm 50 a$. The cylinder is submerged at $h=1.5 a$ (the distance from the centre of the cylinder to the mean free surface). The water depth is taken as $d=4 a$, and $\omega=1.0$. The forces (per unit length) have been nondimensionalized by $\rho \delta_{0} a g$. It has been shown recently by $\mathrm{Wu}(1993)$ that when a cylinder which is symmetric about a vertical plane oscillates horizontally, the horizontal force has components at frequencies $(2 n+1) \omega$ and the vertical force has components at $2 n \omega(n=0,1,2 \ldots)$. This is confirmed in Fig. 4(a) and (b). Figure 4(c) gives the wave elevation at $t=30$. All these figures show that the results from the two methods are in very good agreement.

Figure 5 gives the results for the same problem when the cylinder is in vertical 

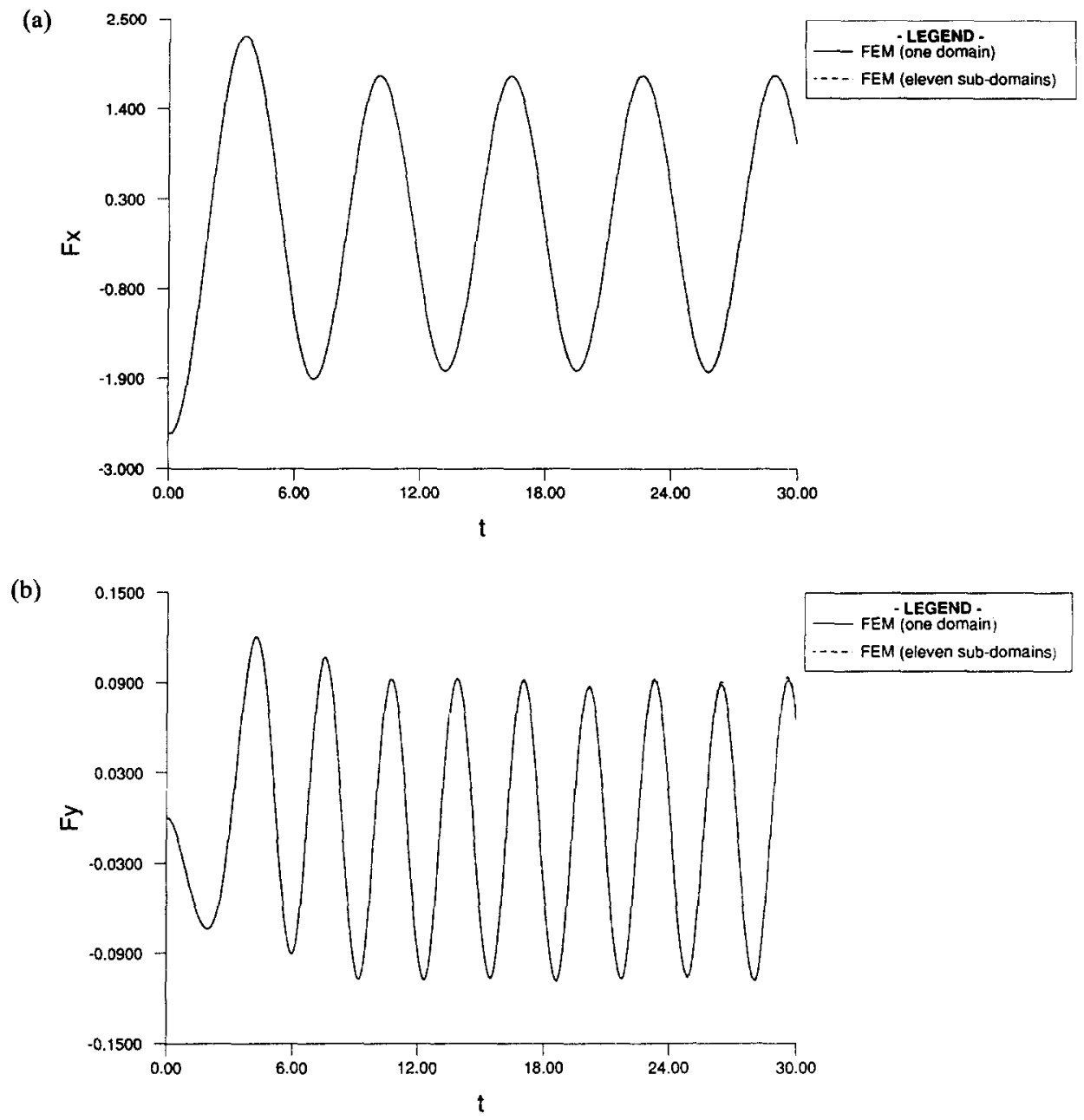

(c)

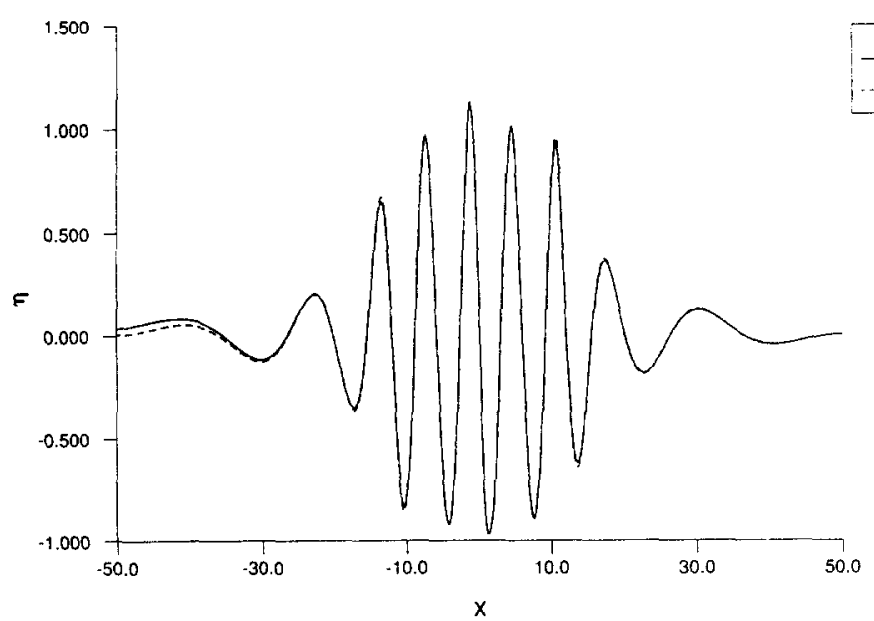

Fig. 6. Results for a submerged cylinder undergoing horizontal motion, based on domain decomposition (a) horizontal force; (b) vertical force; (c) wave elevation at $t=30$. 
motion $(\delta=0)$. The force has been nondimensionalized by $\rho \zeta_{0}$ ag. Since the problem is symmetric about $x=0$ and the horizontal force is zero, only the vertical force is provided in Fig. 5(a). In Fig. 5(b), the wave elevation at $t=30$ is shown. These figures again demonstrate that results from the two methods are in good agreement.

Lastly, we demonstrate use of the domain decomposition method based on the case analysed in Fig. 4. Eleven finite element sub-domains were used: one of length $20 a$ centred on the axis of the cylinder and five on either side each of length $8 a$. At each time step, $\lambda_{0}$ is taken from the solution at the previous step. Equations (21) and (22b) are then repeatedly used until the differences between the potentials at the interfaces of any two adjacent domains are less than $10^{-3}$. Figure 6 gives the comparison of results from the methods with and without domain decomposition. As can be seen they agree very closely.

\section{CONCLUSIONS}

Some two-dimensional nonlinear time domain free surface flow problems have been solved by two numerical methods. It appears from this that in many cases the finite element method may be more efficient and need less memory than the boundary element method. However, this conclusion is so far based on rather limited experience. For a complicated structure or a multibody problem, it is likely that the bandwidth of the matrix in the finite element method may not be easy to control. This will then increase the CPU time and memory required. Another possible disadvantage of the finite element method is that the mesh for a complicated body is not easy to generate. Automated mesh generators and graphics preprocessors, however, are easing this difficulty. Finally, our preliminary results also show that the domain decomposition method provides accurate results, although further work is needed in this direction.

Acknowledgements-This work forms part of the research programme "Uncertainties in Loads on Offshore Structures" sponsored by EPSRC through MTD Ltd and jointly funded with: Amoco (UK) Exploration Company, BP Exploration Operating Co. Ltd, Brown \& Root Ltd, Exxon Production Research Company, Health and Safety Executive, Norwegian Contractors a.s., Shell UK Exploration and Production, Den Norske State Oljeselskap a.s., Texaco Britain Ltd.

\section{REFERENCES}

Glowinski, R., Dinh, Q.V. and Periaux, J. 1984. Domain decomposition for elliptic problems. In Finite Elements in Fluids. John Wiley and Sons, Chichester, Vol. 5, pp. 45-106.

Lin, W.M., Newman, J.N. and Yue, D.K. 1984. Nonlinear forced motion of floating bodies. Proc. 15th Symp. on Naval Hydrodynamics, Hamburg, Germany, pp. 33-47.

Longuet-Higgins, M.S. and Cokelet, E.D. 1976. The deformation of steep surface waves on water, I: a numerical method of computation. Proc. $R$. Soc. Lond. A350, 1-25.

New, A.L. McIver, P. and Peregrine D.H. 1985. Computations of overturning waves. J. Fluid Mech. 150, 233-251.

Newman, J.N. 1977. Marine Hydrodynamics. MIT Press, Cambridge, MA.

Telste, J.G. 1985. Calculation of fluid motion resulting from large-amplitude forced heave motion of a twodimensional cylinder in a free surface. Proc. 4th Int. Conf. on Numerical Ship Hydrodynamics, Washington, U.S.A., pp. 81-93.

Vinje, T. and Brevig, P. 1981. Nonlinear ship motion. Proc. 3rd Int. Conf. on Numerical Ship Hydrodynamics, Washington, U.S.A., PP. 257-266.

Yeung, R.W. and Vaidhanathan, M. 1990. Nonlinear wave diffraction over submerged obstacles. Proc. 5th Int. Workshop on Water Waves and Floating Bodies, Manchester, U.K.

Wu, G.X. 1993. A note on non-linear hydrodynamic forces on a body submerged below a free surface. Appl. Ocean Res. 15, 371-372. 
Wu, G.X. and Eatock Taylor, R. 1994. Finite element analysis of two dimensional non-linear transient waves waves. Appl. Ocean Res. 16, 363-372.

Zhao, R. and Faltinsen, O.M. 1992. Water entry of two dimensional bodies. J. Fluid Mech. 246, 593-612. 